University of Nebraska - Lincoln

DigitalCommons@University of Nebraska - Lincoln

\title{
A Review of Factors and Display Characteristics Influencing P300 Brain-Computer Interface Performance for AAC.
}

Kevin Pitt

University of Nebraska-Lincoln, kevin.pitt@unl.edu

Follow this and additional works at: https://digitalcommons.unl.edu/specedfacpub

Part of the Special Education and Teaching Commons

Pitt, Kevin, "A Review of Factors and Display Characteristics Influencing P300 Brain-Computer Interface Performance for AAC." (2020). Special Education and Communication Disorders Faculty Publications. 220.

https://digitalcommons.unl.edu/specedfacpub/220

This Article is brought to you for free and open access by the Department of Special Education and Communication Disorders at DigitalCommons@University of Nebraska - Lincoln. It has been accepted for inclusion in Special Education and Communication Disorders Faculty Publications by an authorized administrator of DigitalCommons@University of Nebraska - Lincoln. 
Pitt, K. (2020). A Review of Factors and Display Characteristics Influencing P300 Brain-Computer Interface Performance for AAC. Accepted for presentation at the 2020 International Society for Augmentative and Alternative Communication (ISAAC) Conference, Cancun, Mexico. (convention cancelled).

doi: $10.32873 /$ unl.dc.oth.002

https://doi.org/10.32873/unl.dc.oth.002

A Review of Factors and Display Characteristics Influencing P300 Brain-Computer Interface Performance for AAC.

Pitt, K

\begin{abstract}
For individuals with amyotrophic lateral sclerosis (ALS), neurodegeneration can lead to a state of near-total paralysis, with intact cognition and sensation (Fried-Oken. et al., 2013). This loss of voluntary speech and motor system control (including oculomotor) can cause individuals with ALS to lose a reliable form of communication. Brain-computer interfaces for AAC (BCI-AAC) can provide a non-invasive link between an individual's neurologic activity and a communicational device via electroencephalography (EEG), circumventing the necessity of reliable motor control for AAC access. Different BCI techniques may afford communication access through a range of techniques such as the P300 (including rapid serial visual presentation (RSVP) of items), steady state evoked potential, and motor imagery (see Brumberg, Pitt, MantieKozlowski \& Burnison, 2018; and Rezeika et al., 2018, for a full review of BCI techniques).

In the past decade there has been an increased focus into the application of BCI for AAC, with P300-based BCI-AAC devices becoming commercially available (i.e., the P300 Intendix Speller from g.tec medical engineering). However, there are currently limited guidelines regarding how to effectively feature match an individual to an AAC-BCI technique and display (e.g., Pitt, Brumberg \& Pitt., 2019). Similar to existing AAC techniques, the best BCI match for an individual may vary between different types of BCI devices (e.g., P300 versus motor imagery), due to factors including; the highly contrasting user interfaces, and neural signals upon which the BCI functions (see Pitt \& Brumberg, 2018 for review). Therefore, further research is needed to identify factors that may be of important consideration in BCI assessment, especially for visually based P300-based BCI techniques such as the Intendix speller, which will likely to be the first BCIs to enter clinical practice.
\end{abstract}

\begin{abstract}
Aims
To help inform assessment and research considerations for P300-based BCI-AAC devices (including both the P300-grid and P300-RSVP presentation formats), along with educating stakeholders (e.g., AAC professionals, along with individuals using AAC and their communication facilitators) about P300 BCI techniques, through this study we aimed to 1) review current factors influencing P300-based performance, along with 2) exploring how different P300 display characteristics may influence P300-based BCI communication success.
\end{abstract}




\section{Method}

A literature review was performed to identify current research discussing BCI performance predictors, and assessment considerations for P300-based BCIs for communication. Multiple databases were searched including, but not limited to, ERIC, PubMed/MEDLINE, SpringerLink, Plos, \& Google Scholar. Broad search terms (e.g., "P300-based brain-computer interface", plus "assessment" or "screening" or "alternative and augmentative communication"). Broad terms were used to ensure relevant articles were not missed. Due to the increased proliferation in AAC$\mathrm{BCI}$ research over the past decade; dates between 2000 to present were incorporated.

\section{Results:}

For this abstract reference are not provided in the results section to meet ISAAC submission requirements which only allow submissions to include a maximum of six references. However, full references will be provided in the presented product. In addition, the presented product will include details for each identified area outlined below, along with a review of how P300-based BCIs acquire and decode the target signal for translation into BCI-based communication output (e.g., through the P300 'oddball' presentation paradigm; Brumberg et al., 2018).

A) Factors: The literature review revealed a range of factors which may influence P300based BCI performance, including:

- Degree of oculomotor (eye) control

- Working memory

- Sustained attention

- Visual Perception

- Level of concentration

- Neurophysiological (e.g., increased P300 amplitude, compression of posterior recording electrodes)

- Workload and fatigue

- Amount of food consumption

- Mood and motivation

- Medical history (e.g., pharmaceuticals, history of seizures)

- Sources of noise (e.g., from the environment, uncontrolled movements).

B) Display Characteristics: Similar to existing AAC techniques (e.g., Light, Wilkinson, Thiessen, Beukelman, \& Fager, 2019), changes in display design may influence P300-based BCI outcomes. For instance, RSVP presentation which display items from a single (e.g., central) screen location may support those with oculomotor difficulties, and the use of faces to highlight images during the P300 oddball paradigm may improve P300-based BCI performance, and possibly support BCI success for those with cognitive impairments. Further, the use of color may also see to support BCI performance (e.g., green faces, highlighting grid letters in color versus black and white), and motion may also be used to draw attention to the BCI interface (e.g., letters move from side to side).

\section{Conclusion:}


The information generated from this study will help educate stakeholders about P300-based BCI techniques, along with informing $\mathrm{AAC}-\mathrm{BCI}$ development, and assessment considerations for helping accurately feature match an individual to a communicational $\mathrm{BCI}$ devices.

\section{References}

Brumberg, J., Pitt, K., Mantie-Kozlowski, A., \& Burnison, J. (2018). Brain-Computer Interfaces for Augmentative and Alternative Communication: A Tutorial. American Journal of Speech-Language Pathology, 27, 1-12

Fried-Oken, M., Mooney, A., Peters, B., \& Oken, B. (2013). A clinical screening protocol for the RSVP Keyboard brain-computer interface. Disability and Rehabilitation: Assistive Technology, 10(1), 11-18.

Light, J., Wilkinson, K. M., Thiessen, A., Beukelman, D. R., \& Fager, S. K. (2019). Designing effective AAC displays for individuals with developmental or acquired disabilities: State of the science and future research directions. Augmentative and Alternative Communication, 35(1), 42-55.

Pitt, K., \& Brumberg, J. S. (2018). Guidelines for Feature Matching Assessment of BrainComputer Interfaces for Augmentative and Alternative Communication. American Journal of Speech-Language Pathology, 27, 950-964.

Pitt, K., Brumberg, J. \& Pitt, A. (2019). Considering Augmentative and Alternative Communication Research for Brain-Computer Interface Practice. Assistive Technology Outcomes and Benefits, 13., 1-19.

Rezeika, A., Benda, M., Stawicki, P., Gembler, F., Saboor, A., \& Volosyak, I. (2018). BrainComputer Interface Spellers: A Review. Brain Sciences, 8(4), 57

Learning objectives

1) Discuss the basics of how P300-based BCIs for AAC function.

2) Describe different person-centered factors that may influence P300-based BCI-AAC success

3) Discuss how P300-based BCI-AAC display characteristic may be modified.

\section{Author details}

Name

Institution

Address

Phone/fax/email

Biographical Sketch
Kevin Pitt, PhD., CCC-SLP

University of Nebraska-Lincoln

357 Barkley Memorial Center, Lincoln, NE, 68583

Kevin.pitt@unl.edu

Kevin Pitt is an assistant professor in Communication

Sciences and Disorders at the University of Nebraska-

Lincoln, and PI of the AAC Translation (AACT)

Laboratory. He is interested in translating the latest AAC 
access advancements such as brain-computer interfaces out of the laboratory setting by incorporating current clinical procedures, stakeholder input, and principles governing display design. 